\title{
Floating Aortic Thrombus: A Rare Cause of Acute Ischemic Stroke Necessitating Modification of Access Route for Thrombectomy
}

\author{
Sinan Balci, MD, ${ }^{*}$ Ethem Murat Arsava, $\mathrm{MD}, \dagger$ \\ Mehmet Akif Topcuoglu, MD, + and Anil Arat, MD*
}

\begin{abstract}
We report 2 patients with acute occlusion of middle cerebral artery successfully treated by mechanical thrombectomy performed via transbrachial access. Both patients had floating aortic arch thrombi precluding safe transfemoral access due to risk of further iatrogenic embolization. Moreover both patients were diagnosed with hypercoagulopathy secondary to lung malignancy (paraneoplastic etiology, Trousseau's syndrome) as the cause of both aortic thrombi and acute ischemic stroke. Mechanical thrombectomy in the setting of a floating aortic thrombus has been mentioned only once as part of general management of floating aortic thrombi in the whole body. To the best of our knowledge, it has never been described previously in the literature in the context of cerebral mechanical thrombectomy technique. However, the diagnosis of this entity bears clinical importance for it can modify the treatment approach. A modified transbrachial approach allowed us to treat both patients without neurologic complications and resulted in modified Rankin scores of 1 on follow-up. We advise that the cross-sectional imaging of acute stroke patients should include an evaluation of the aortic arch and should be scrutinized in detail especially in patients with possible hypercoagulable state. Flat panel computed tomography technology allowed us to obtain such an imaging study in the angiography suite.

Key Words: Acute ischemic stroke-mechanical thrombectomy-transbrachial access-aortic thrombus-protruding aortic thrombus

(c) 2019 Elsevier Inc. All rights reserved.
\end{abstract}

\section{Introduction}

Endovascular technique of thrombectomy, the current standard of treatment in large vessel occlusion in the setting of acute ischemic stroke (AIS), is well defined, ${ }^{1}$ however modifications of the technique, including modification of

From the *Department of Radiology, Hacettepe University Faculty of Medicine, Ankara, Turkey; and +Department of Neurology, Hacettepe University Faculty of Medicine, Ankara, Turkey.

Received April 12, 2019; revision received May 27, 2019; accepted July 10, 2019.

Financial Disclosure: No funding or grant is received.

Address correspondence toAnil Arat, MD, Department of Radiology, Hacettepe University Faculty of Medicine, Sihhiye, 06100 Ankara, Turkey. E-mail: anilarat@hotmail.com.

1052-3057/\$ - see front matter

(c) 2019 Elsevier Inc. All rights reserved.

https:/ / doi.org/10.1016/j.jstrokecerebrovasdis.2019.07.007 endovascular access site may be necessary in unusual presentations of AIS. Herein we present 2 cases of successful mechanical thrombectomy procedures in which conventional transfemoral access was deemed to be risky because of mural aortic arch thrombi, which were considered as the cause of AIS. Both patients had a prothrombotic state secondary to an underlying lung carcinoma, and were treated safely via a right transbrachial access (TBA). Endovascular treatment (EVT) of AIS secondary to an aortic thrombus has been mentioned, without description of the EVT technique used, in only 2 cases previously. ${ }^{2}$ Although transbrachial or transradial access for EVT of AIS has been reported recently, ${ }^{3-5}$ the choice of the alternative access was based on operator preference and in many cases due to expected delays in recanalization had transfemoral access been used. Our patients are the first, to our knowledge, in whom an 
alternative access was mandated because of the risk of further embolization from aortic thrombus.

\section{CASE 1}

A 57-year-old man presented to the emergency department of our hospital with loss of consciousness, rightsided hemiplegia and aphasia with a symptom onset-todoor time of 2 hours. His admission NIHSS score was 17. His clinical history was remarkable for coronary artery disease and metastatic lung cancer. Head and neck computed tomography angiography (CTA) demonstrated left Middle cerebral artery (MCA) M1 segment occlusion as well as a $2.5 \times 1 \mathrm{~cm}$ thrombus protruding into the aortic arch from origin of left subclavian artery (LSCA) causing LSCA occlusion and extending to the origin of the left common carotid artery (CCA) (Fig 1). Other CTA findings included a spiculated mass lesion in apical zone of the left lung and accompanying fibrotic changes next to mediastinum consistent with the history of radiotherapy. He did not receive intravenous rt-PA prior to the procedure since left frontal and right cerebellar hyperdense metastatic lesions were noted on initial noncontrast head CT. Due to a potential hazard of dislodging the aortic thrombus during catheterization and causing further embolic events, we opted to gain intraarterial access via the right brachial artery instead of the common femoral artery for thrombectomy. Under general anesthesia, systemic anticoagulation and ultrasonographic guidance the right brachial artery was punctured at the level of midlower arm via 21 gauge micropuncture needle. After placement of a short 6 Fr sheath, instead of using a Simmons-2 type of catheter

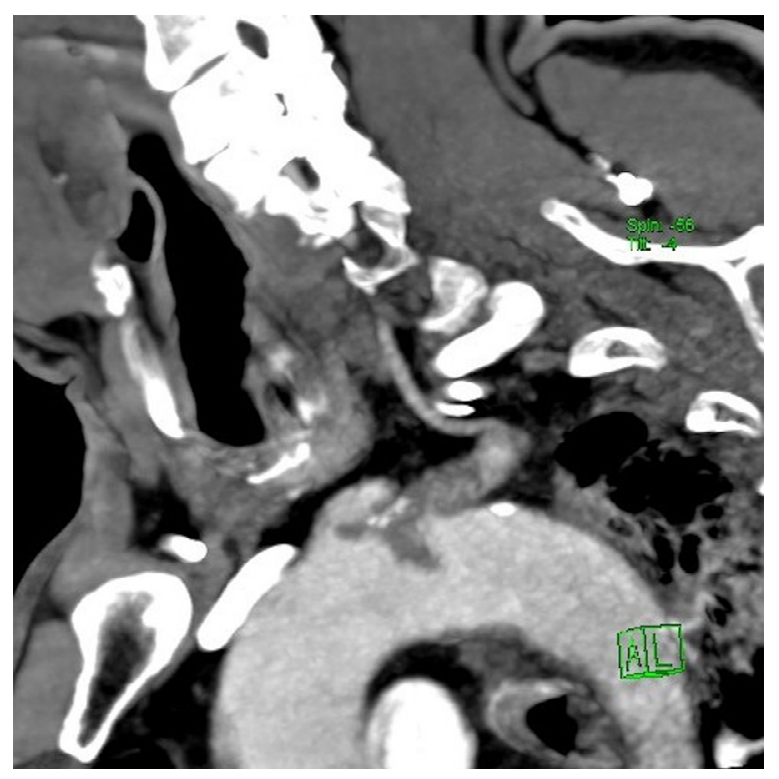

Figure 1. Occlusion of left subclavian artery origin and floating component of thrombus in the aortic arch next to the left CCA origin are seen on oblique maximum intensity projection reformatted neck CT angiography image. Note that the origin of the left vertebral artery is patent. Abbreviations: CCA, common carotid artery. as previously described for transradial/ $\mathrm{TBA}^{3}$ we opted to use a Simmons- 1 catheter based on the morphology of the aortic arch on CTA. The catheter was directly advanced to the right subclavian artery and the vertebral artery was directly selected so that the curve of the catheter was located at the origin of the vertebral artery. A vertebral angiogram was obtained to rule out a vertebrobasilar clot secondary to the left subclavian thrombus, which demonstrated a left subclavian steal. Then the guidewire was advanced as far as the curve of the catheter and the catheter was simply pushed forward for it to assume the regular Simmons-1 shape. The catheter directly engaged the left CCA origin (Fig 2). The short 6F sheath was exchanged with a 6 Fr $45 \mathrm{~cm}$ long Pinnacle Destination guiding sheath (Terumo Medical, Somerset, NJ) which was navigated to the origin of the left CCA with its tip pointing upward and providing support for a 5 Fr Sofia catheter (MicroVention, Tustin, CA) that was navigated over a Rebar 27 microcatheter (ev3, Irvine, CA) and Avigo microwire (ev3, Irvine, CA) to engage the MCA thrombus. After performing a direct aspiration first pass technique (ADAPT) twice, control angiograms showed thrombolysis in cerebral infarction $2 \mathrm{~B}$ recanalization of the left MCA territory with a small residual filling defect in a temporoparietal branch (Fig 3). The symptom to recanalization time was 4 hours. A flat-panel CT of the head revealed no evidence of an intracranial hemorrhage. A flat-panel CT of the arcus aorta obtained during breathhold and with injection of $100 \%$ contrast (iohexol 300, Omnipaque; GE Healthcare, Milwaukee, WI) (42 cc at a rate of $2 \mathrm{cc} / \mathrm{s}$ ) showed no change in the size or configuration of the hanging thrombus at aortic arch (Fig 4). A brachial angio-

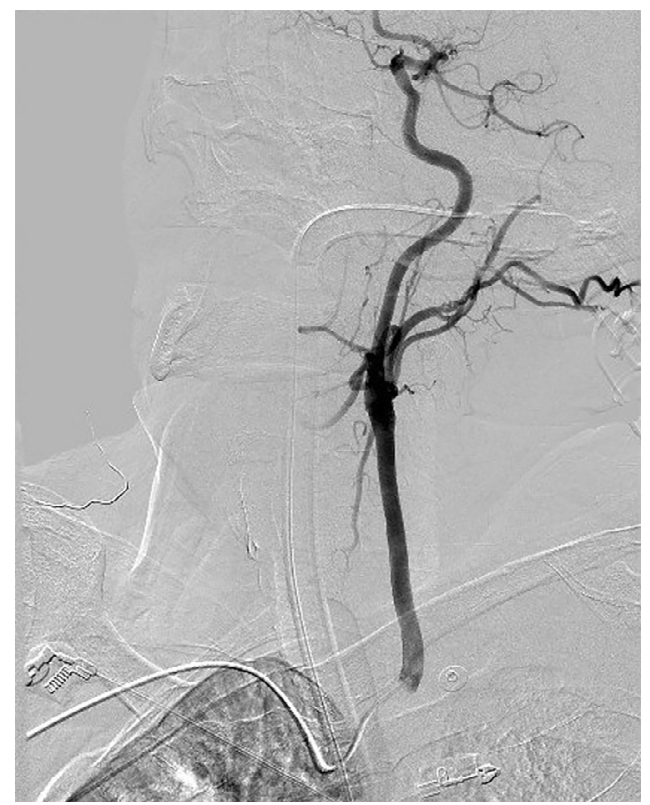

Figure 2. Left CCA angiogram on anteroposterior projection via Simmons1 catheter demonstrates left MCA M1 segment occlusion. Abbreviations: CCA, common carotid artery. 


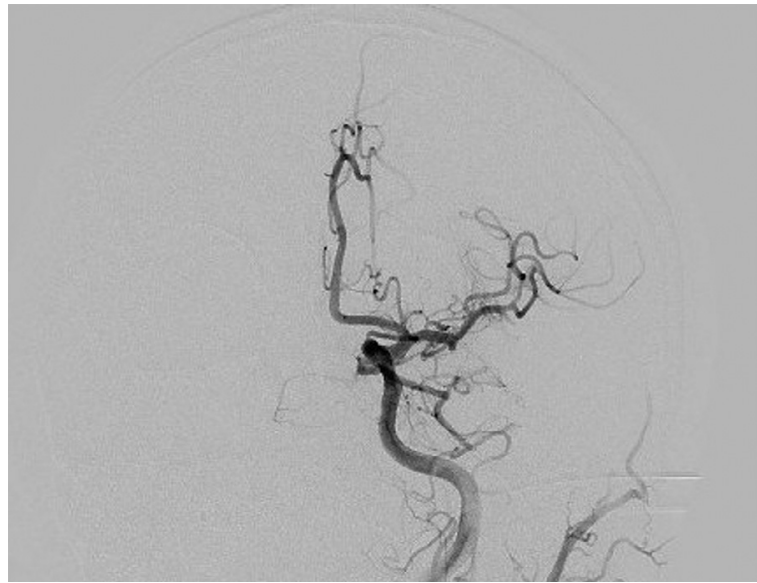

Figure 3. Anteroposterior left CCA angiogram performed after mechanical thrombectomy reveals revascularization of left MCA territory. Abbreviations: CCA, common carotid artery.

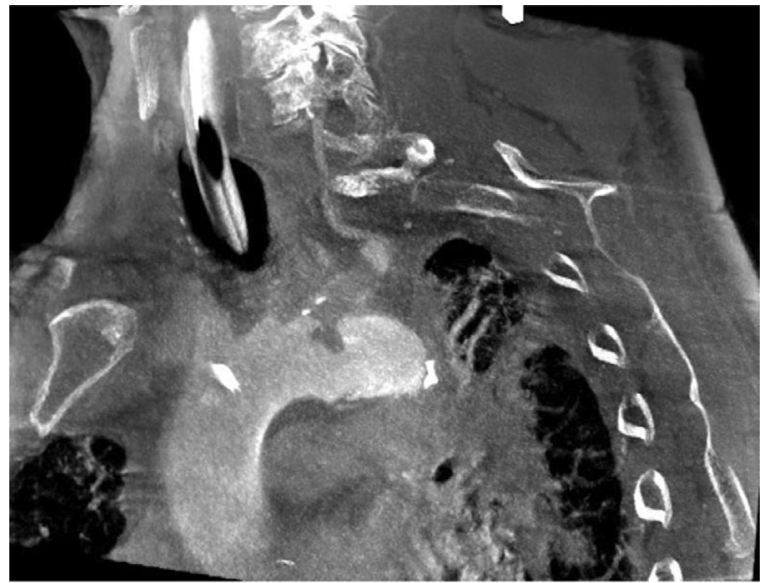

(a)

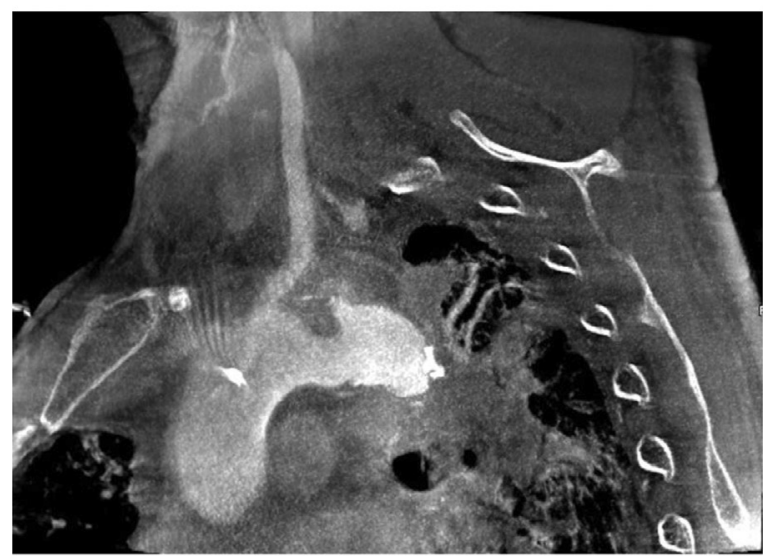

(b)

Figure 4. Maximum intensity projection reformatted flat panel CT images of aortic arch obtained in angiography suite after mechanical thrombectomy show left subclavian artery occlusion (a) and stable floating thrombus next to the left CCA origin (b). Note spiculated mass and accompanying fibrotic changes in left lung due to previous radiotherapy in (b). Abbreviations: $C C A$, common carotid artery.

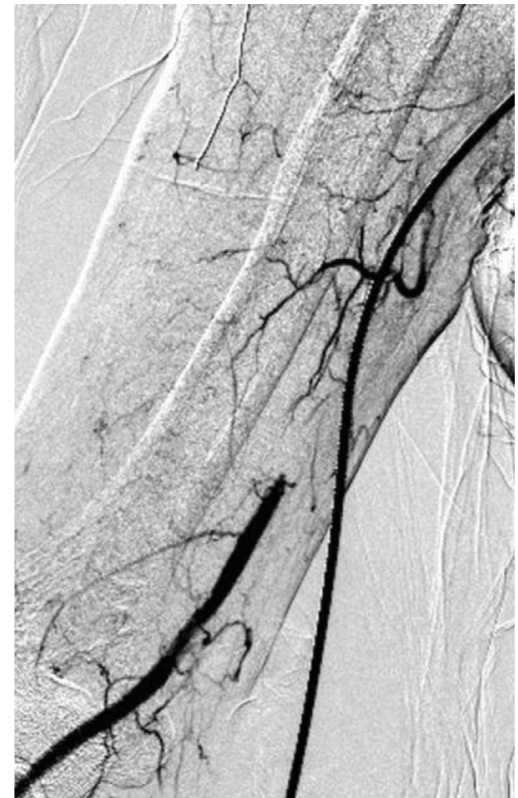

Figure 5. Right brachial angiogram performed at the end of the operation shows segmental occlusion of the right brachial artery and accompanying distal vascularization via collaterals.

gram showed occlusion of the right brachial artery with good collateralization of the arm and hand (Fig 5). There was no clinical evidence of right hand ischemia. Local hemostasis at the arterial puncture site was achieved with manual compression. During and after the procedure the mean blood pressure as measured from the right arm was approximately $90 \mathrm{~mm} / \mathrm{Hg}$; mean blood pressure measured from the left arm was approximately $20 \mathrm{~mm} / \mathrm{Hg}$ lower. The patient had no symptoms attributable to the left subclavian steal hence no treatment was offered. The patient was kept on systemic anticoagulation during his hospital stay. On postoperative day 4, CTA of the head and neck showed that the aortic component of the thrombus had resolved and the LSCA remained occluded. On postoperative day 5 , the day of discharge, the NIHSS score of patient was 4. Pathological analysis of the retrieved thrombi revealed blood elements and fibrin. At 2 months, his modified Rankin score 1 and he only had minimal aphasia permitting almost complete communication.

\section{CASE 2}

A 60-year-old man presented to emergency department of our hospital with acute onset left hemiplegia with a symptom onset-to-door time of 1.5 hours. His admission NIHSS score was 16 . His clinical history was remarkable for metastatic lung cancer and recent systemic chemotherapy. Head and neck CTA demonstrated right MCA M1 segment occlusion as well as central spiculated mass and accompanying atelectatic areas in right lung and there were 4 different foci of mural thrombi in the aortic arch (Fig $6 \mathrm{a}-\mathrm{c}$ ). He did not receive intravenous rt-PA because 


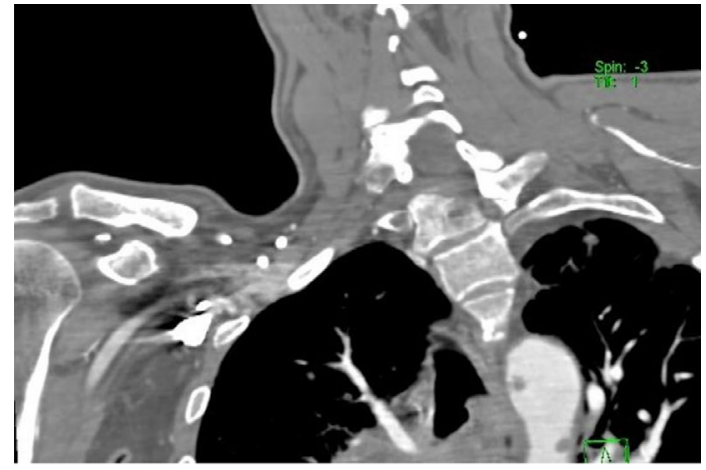

(a)

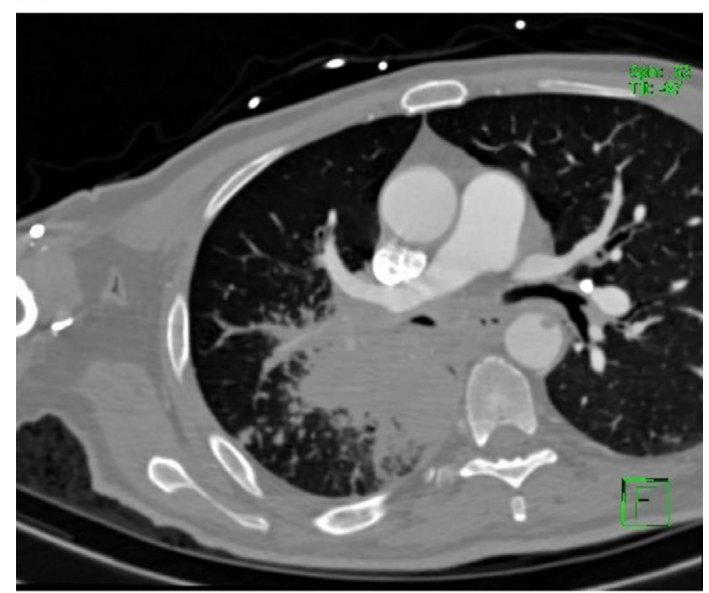

(b)

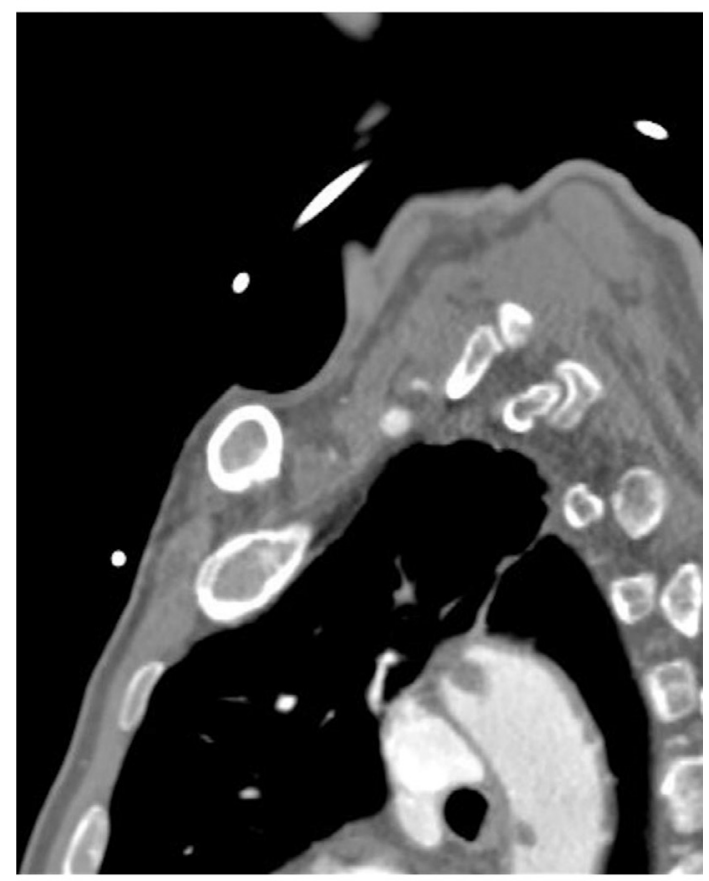

(c)

Figure 6. Oblique coronal (a), axial (b), and sagittal (c) reformatted neck $C T$ angiography images reveal a central spiculated mass in right lung as well as small mural thrombi in aortic arch. of a left cerebellar well circumscribed hypodense area of approximately $2 \mathrm{~cm}$ which was suspicious of metastasis on initial noncontrast head CT. Due to a potential hazard of dislodging the thrombi during catheterization via transfemoral approach and causing further embolic events, we opted to gain intraarterial access via right arm for thrombectomy. Under general anesthesia, systemic anticoagulation and ultrasonographic guidance the right brachial artery was punctured at the level of midlower arm via 21 gauge micropuncture needle. After infusion of $0.4 \mathrm{mg}$ nimodipine and placement of 6 Fr $45 \mathrm{~cm}$ long Pinnacle Destination guiding sheath (Terumo Medical, Somerset, NJ), a 6F Simmons Select catheter (Penumbra) was navigated up to the right cervical ICA. A 5 Fr Sofia catheter (MicroVention, Tustin, CA) was navigated through the sheath over a Rebar 27 microcatheter (ev3, Irvine, CA) and Avigo microwire (ev3, Irvine, CA) to engage the MCA thrombus and it was pulled back during aspiration (Fig 7a and b). After performing this procedure once, control angiograms showed thrombolysis in cerebral infarction $2 \mathrm{~B}$ recanalization of right MCA territory with a residual filling defect in a distal branch (Fig 8a and b). The symptom to recanalization time was 3 hours. A flat-panel CT of the head revealed no evidence of an intracranial hemorrhage. A brachial angiogram showed moderate focal spasm at the entry site (Fig 9). Local hemostasis at the arterial puncture site was achieved with manual compression. At discharge on day 5, the NIHSS score of patient was 5. Pathological analysis of the retrieved thrombi disclosed only blood elements and fibrin. At 1 month, the patient was modified Rankin score 1.

\section{Discussion}

Focal aortic thrombus (so called protruding or floating aortic thrombus) is an underestimated source of distal emboli. ${ }^{2}$ AIS secondary to such a thrombus has been rarely documented in the literature ${ }^{2,6}$ and it was reported to account for up to $2.5 \%$ of all AIS cases in a recent study. ${ }^{7}$ Since floating aortic thrombus is a rarely encountered and reported entity, standard of care for either the thrombus itself or the secondary AIS has not been well defined. Surgical thrombectom $y^{2}$ and systemic anticoagulation $^{8}$ for thrombus removal have been reported as therapeutic options depending on the clinical status. In our first case, part of the floating thrombus in the aortic arch resolved with systemic anticoagulation within 1 week. Given that no other underlying source of thromboembolism was demonstrated in spite of a detailed work-up, the most plausible explanation of the AIS in our both cases was in situ thrombosis in aorta as part of a paraneoplastic hypercoagulable state (Trousseau's syndrome). Floating aortic thrombus is regarded as a separate clinical entity from atherosclerotic disease since development of a 


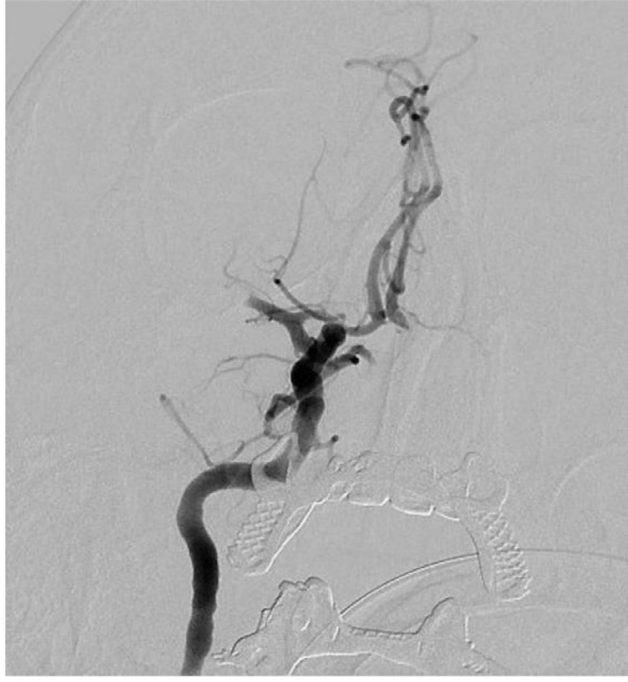

(a)

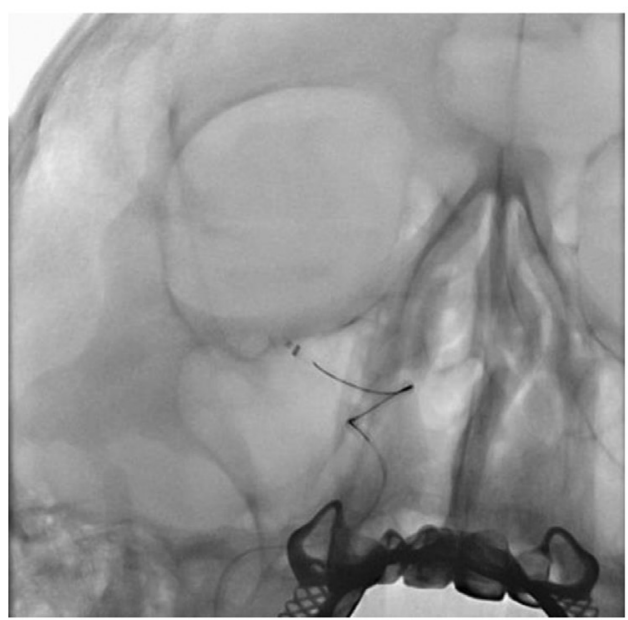

(b)

Figure 7. Cutoff in proximal main truncus of right MCA consistent with thrombosis is shown on anteroposterior right internal carotid artery angiogram (a). Engagement of the clot via aspiration catheter navigated over a microcatheter is seen (b).

thrombus in aortic arch requires additional underlying pathologic states such as hypercoagulability syndromes, collagen tissue diseases or malignancy ${ }^{2,9}$ which is also the case in our patients. In the literature we were able to find 2 cases of mechanical thrombectomy in one of the largest series focusing on the treatment of aortic floating thrombus $^{2}$ in general, including those in the descending and abdominal aorta. The authors of this series mention about some form of EVT in one of their patients but provide no information as to either the procedure or the neurologic outcome since the study is not related to cerebral consequences of aortic thrombus. Apart from this we were unable to find a description of EVT in AIS due to an aortic thrombus. ${ }^{2}$

With regard to access site, mechanical thrombectomy via transradial (TRA) and/or TBA in AIS patients has been recently reported in literature for both the anterior

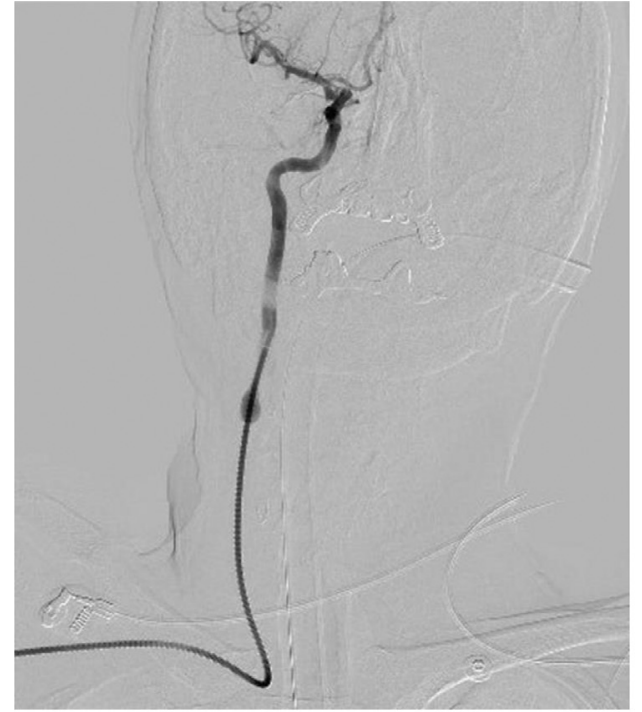

(a)

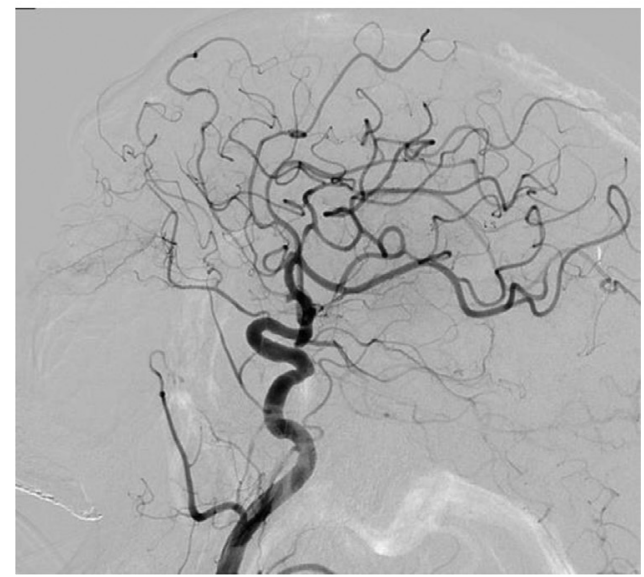

(b)

Figure 8. Postprocedural angiograms demonstrate TICI $2 B$ revascularization of the right MCA territory. Abbreviations: TICI, thrombolysis in cerebral infarction.

and posterior circulations. ${ }^{10,11}$ In some cases transradial/ transbrachial route is chosen as initial access based on cross-sectional imaging findings ${ }^{11}$ or history of femoral artery surgery ${ }^{5}$; meanwhile in some cases operators shifted to arm access after unsuccessful catheterization via transfemoral route. ${ }^{5,12}$ Typically, TRA and TBA are preferred when the transfemoral approach fails in anterior circulation AIS which happens in about $0.5 \%$ of patients. ${ }^{13}$

Reviewing the literature, we encountered three series of TRA with a total of 54 cases $^{3,4,10}$ and only 9 cases of TBA for the treatment of AIS. ${ }^{5,12,14}$ In all of these reported AIS cases treated through a TBA or TSA, the reason for switching to an alternate route was either operator preference (TRA as the default access path) ${ }^{4}$ or hardship in accessing the target artery via a transfemoral route. ${ }^{5,10,12}$ The reason for choosing TBA in our 


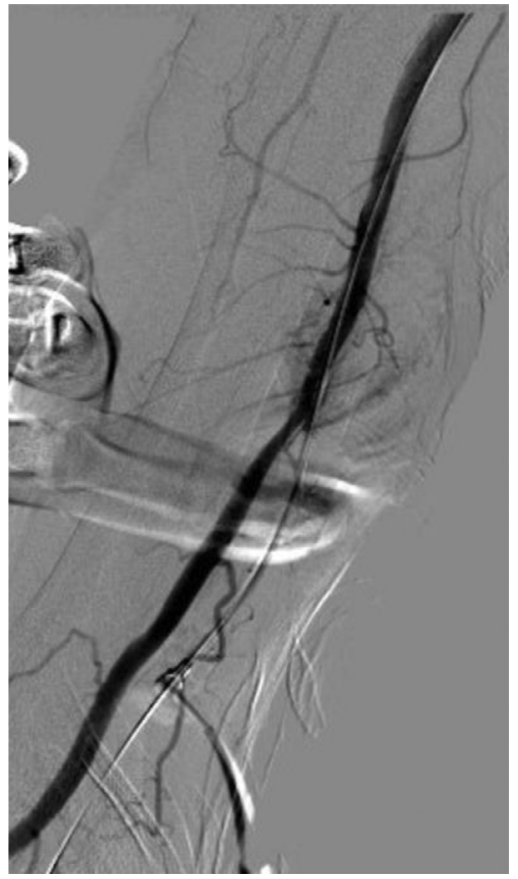

Figure 9. Right brachial angiogram performed at the end of the operation reveals minimal focal spasm at the entry site with normal distal vascularization.

cases was not the inability to reach left CCA but the potential danger of embolization from the aortic thrombus with transfemoral access. Consequently, our 2 patients represent the only cases in our practice who needed an alternative access for thrombectomy for AIS treatment. They are also the first cases in the literature in whom switching to an alternative access was mandated by aortic pathology, stressing on the fact that neurointerventionalists need to be familiar with alternative access routes because an urgent need for such access may come up during AIS treatment.

Most of the previous reports on thrombectomy with TBA/TRA employed large-bore (0.070-0.088 inch) guide catheters which were advanced over a 5 Fr Simmons-2 catheter. ${ }^{3-5}$ Various catheter maneuvers were performed to shape the long catheter. ${ }^{3}$ We used a $100 \mathrm{~cm}$ long Simmons-1 catheter which is easier to shape, without a need to maneuver the catheter in the aorta. We chose this catheter in our case because we wanted to refrain from catheter manipulations in the aorta and endanger embolization of the floating aortic thrombus. It is possible to shape this catheter within the subclavian artery and the maneuver is much simpler than that for a Simmons-2 catheter. In addition, unlike the previous reports, we used a sheath with a tip shape which are, in general used to prevent prolapse of stents and balloons into the aorta during renal interventions. As we expected, in our cases it enabled us to have better support without falling back into the aorta and providing support for navigation of an aspiration catheter intracranially.
There are both advantages and disadvantages of a brachial approach which has been described in 3 reports of a total of 9 cases to date. ${ }^{5,12,14}$ The disadvantage, as compared to the TRA is that the brachial artery is an "end artery" functionally so its occlusion may result in upper extremity ischemia. As unpleasant and undesirable as this may be, there exists a surgical treatment for this condition which can be performed directly after intracranial thrombectomy. We also believe that achieving hemostasis is harder in TBA as compared to TRA. That being said, the brachial artery is larger than the radial artery, with a lower likelihood of occlusion, especially when intraarterial nimodipine is injected and the procedure is performed under anticoagulation. Another advantage is that due to the shorter distance from the origins of the supraaortic major trunks, it allows the operator to use a variety of shorter sheaths. Among patients with failed access for intracranial interventions, 2 other methods exist; transcervical (direct carotid puncture) ${ }^{15,16}$ and the recently described transvenous/transseptal access. ${ }^{17}$ However serious consequences such as neck hematoma and subsequent compromise of airway may be associated with direct carotid puncture. ${ }^{15}$ Transvenous/transseptal access is logistically harder to perform in an emergency condition and would be of no use in our patients as it would still entail some catheter manipulation in the aorta.

In conclusion, transbrachial approach can be a feasible alternative route in AIS patients with anterior system occlusion especially when there are factors precluding transfemoral access as well as safe and prompt catheterization of target vessels. Floating aortic thrombus should be kept in mind as a source of AIS in those patients with a tendency for thrombosis.

\section{Declaration of Competing Interest}

No conflict of interest.

\section{References}

1. Elgendy IY, Kumbhani DJ, Mahmoud A, et al. Mechanical thrombectomy for acute ischemic stroke: a meta-analysis of randomized trials. J Am Coll Cardiol 2015;66: 2498-2505.

2. Weiss S, Buhlmann R, von Allmen RS, et al. Management of floating thrombus in the aortic arch. J Thorac Cardiovasc Surg 2016;152:810-817.

3. Snelling BM, Sur S, Shah SS, et al. Transradial approach for complex anterior and posterior circulation interventions: technical nuances and feasibility of using current devices. Oper Neurosurg 2018;17:293-302.

4. Sur S, Snelling B, Khandelwal P, et al. Transradial approach for mechanical thrombectomy in anterior circulation large-vessel occlusion. Neurosurg Focus 2017;42:E13.

5. Okawa M, Tateshima S, Liebeskind D, et al. Successful recanalization for acute ischemic stroke via the transbrachial approach. J Neurointerv Surg 2016;8:122-125.

6. den Uil SH, Vermeulen EGJ, Metz R, et al. Aortic arch thrombus caused by nitrous oxide abuse. J Vasc Surg Cases Innov Tech 2018;4:80-82. 
7. Harloff A, Handke M, Reinhard M, et al. Therapeutic strategies after examination by transesophageal echocardiography in 503 patients with ischemic stroke. Stroke 2006;37:859-864.

8. Stollberger C, Kopsa W, Finsterer J. Resolution of an aortic thrombus under anticoagulant therapy. Eur J Cardiothorac Surg 2001;20:880-882.

9. Toyama M, Nakayama M, Hasegawa M, et al. Direct oral anticoagulant therapy as an alternative to surgery for the treatment of a patient with a floating thrombus in the ascending aorta and pulmonary embolism. J Vasc Surg Cases Innov Tech 2018;4:170-172.

10. Haussen DC, Nogueira RG, DeSousa KG, et al. Transradial access in acute ischemic stroke intervention. J Neurointerv Surg 2016;8:247-250.

11. Oselkin M, Satti SR, Sundararajan SH, et al. Endovascular treatment for acute basilar thrombosis via a transradial approach: initial experience and future considerations. Interv Neuroradiol 2018;24:64-69.

12. Yamaguchi S, Horie N, Morofuji Y, et al. Rapid recanalization using TrevoProVue through a 4.2 Fr catheter without a guiding catheter via transbrachial approach: a case report. NMC Case Rep J 2017;4:97-99.

13. Leischner H, Flottmann F, Hanning U, et al. Reasons for failed endovascular recanalization attempts in stroke patients. J Neurointerv Surg 2018;11:439-442.

14. Ishikawa O, Tsutsumi K, Yoshikawa G, et al. Thrombectomy using a method to directly insert an aspiration catheter into a 6-Fr sheath-introducer placed into the brachial artery. Jf Neuroendovasc Ther 2018;12: 456-462.

15. Blanc R, Piotin M, Mounayer C, et al. Direct cervical arterial access for intracranial endovascular treatment. Neuroradiology 2006;48:925-929.

16. Roche AD, Murphy B, Adams N, et al. Direct common carotid artery puncture for endovascular treatment of acute large vessel ischemic stroke in a patient with aortic coarctation. J Stroke Cerebrovasc Dis 2017;26: e211-e2e3.

17. Orlov K, Arat A, Osiev A, et al. Transvenous treatment of carotid aneurysms through transseptal access. World Neurosurg 2019;124:459-463. 